\title{
Inferior vena cava filters in cancer patients: to filter or not to filter
}

This article was published in the following Dove Press journal:

Therapeutics and Clinical Risk Management

9 March 2011

Number of times this article has been viewed

\author{
Hikmat Abdel-Razeq' \\ Asem Mansour ${ }^{2}$ \\ Yousef Ismael' \\ Hazem Abdulelah' \\ 'Department of Internal Medicine, \\ 2Department of Radiology, \\ King Hussein Cancer Center, \\ Amman, Jordan
}

Correspondence: Hikmat Abdel-Razeq Department of Internal Medicine, King Hussein Cancer Center, Amman II I94, Jordan

Tel +96265300460 ext I000

Email habdelrazeq@khcc.jo
Purpose: Cancer and its treatment are recognized risk factors for venous thromboembolism (VTE); active cancer accounts for almost $20 \%$ of all newly diagnosed VTE. Inferior vena cava (IVC) filters are utilized to provide mechanical thromboprophylaxis to prevent pulmonary embolism (PE) or to avoid bleeding from systemic anticoagulation in high-risk situations. In this report, and utilizing a case study, we will address the appropriate utilization of such filters in cancer patients.

Methods: The case of a 43-year-old female patient with rectal cancer, who developed deep vein thrombosis following a complicated medical course, will be presented. The patient was anticoagulated with a low molecular weight heparin, but a few months later and following an episode of bleeding, an IVC filter was planned. Using the PubMed database, articles published in English language addressing issues related to IVC filters in cancer patients were accessed and will be presented.

Results: Many recent studies questioned the need to insert IVC filters in advanced-stage cancer patients, particularly those whose anticipated survival is short and prevention of PE may be of little clinical benefit and could be a poor utilization of resources.

Conclusion: Systemic anticoagulation can be safely offered for the majority of cancer patients. When the risk of bleeding or pulmonary embolism is high, IVC filters can be utilized. However, placement of such filters should take into consideration the stage of disease and life expectancy of such patients.

Keywords: anticoagulation, bleeding, chemotherapy

\section{Case presentation}

Our patient is a 43-year-old woman who was first diagnosed with rectal cancer seven years ago. She was treated with neoadjuvant chemotherapy and radiation therapy that lasted three months following which she underwent low anterior resection with ileostomy that was followed by adjuvant chemotherapy. Following the completion of her chemotherapy she underwent secondary anastomosis (ileoanal Hartmann's pouch anastomosis).

Two years later, she had disease recurrence in retroperitoneal lymph nodes and was treated by chemotherapy. A month after stopping treatment, she was found to have further disease progression manifested by new sacral mass and more retroperitoneal lymph nodes. At this time, radiotherapy to the sacral mass was given, and chemotherapy was restarted and continued for six months. Five weeks later, she presented with left lower limb pain, swelling, and redness; iliofemoral deep vein thrombosis (DVT) was confirmed by Doppler ultrasound for which she was put on therapeutic doses of 
low-molecular-weight heparin (LMWH). Disease evaluation at this time showed disease progression in para-aortic lymph nodes and new right hydronephrosis. She had a double $\mathrm{J}$ catheter placed, and additional chemotherapy was started, with no success, however, in controlling her disease. Another oral chemotherapy regimen failed too, with imaging studies showing a new adrenal metastasis and a large destructive mass lesion involving the S1-S2 segment compressing the left nerve roots.

Given the poor response to many lines of chemotherapy and the poor outcome associated with her disease, she was transferred to palliative care for best supportive care. The patient's overall prognosis was discussed with her in details, and she elected to have a "do not resuscitate" (DNR) status.

A few weeks later, she was admitted in order to control her increasing pain with intravenous narcotic infusion. During her hospital stay and while on LMWH, she developed hematuria; urine analysis showed numerous red blood cells and 20 white blood cells, and the urine culture was positive for Escherichia coli for which she was treated with antibiotics. Microscopic hematuria continued, and the primary team planned insertion of an inferior vena cava (IVC) filter.

\section{Discussion}

Venous thromboembolism (VTE), which represents a spectrum of diseases including DVT and pulmonary embolism (PE), occurs more frequently in cancer patients. Cancer and its treatment are recognized risk factors for VTE; some studies have reported a six-fold increased risk of VTE in cancer patients compared with those without. ${ }^{1}$ Active cancer accounts for almost $20 \%$ of all new VTE events occurring in the community. ${ }^{2}$ The risk varies by cancer type, and is especially high among patients with malignant brain tumors and adenocarcinoma of the ovary, pancreas, colon, stomach, lung, prostate, and kidney. ${ }^{3}$

Treatment of VTE typically includes initial anticoagulation with unfractionated heparin, a low molecular weight heparin, or a pentasaccharide-like fondaparinux, ${ }^{4}$ along with vitamin $\mathrm{K}$ antagonists like warfarin. Thrombolytic agents may be used in severe cases. Occasionally, specific clinical situations present in which the risk of PE is very high or systemic anticoagulation may be associated with high risk of bleeding; in these instances, IVC filters are utilized to provide mechanical thromboprophylaxis to prevent $\mathrm{PE}$, the life-threatening complication of VTE. Such filters are inserted using a relatively noninvasive technique to maintain central flow. Thanks to newer technology, the IVC filters are becoming a very attractive option and can function with anticoagulation to optimize the prophylaxis strategy.

Inferior vena cava filters are usually utilized in many clinical situations (Table 1). ${ }^{5,6}$ However, many of these indications are subjective, and consensus may occasionally be difficult to reach. In a community-based study, researchers at McMaster University reviewed 1547 local county residents with confirmed diagnosis of acute VTE and without a prior IVC filter. Following the VTE, 203 (13.1\%) patients had an IVC filter placed. In reviewing the indications for IVC filter placement, panel members unanimously agreed that the use of an IVC filter was appropriate in $51 \%$ of the cases and inappropriate in $26 \%$; no consensus was reached in the remaining $23 \%$ of the cases. ${ }^{7}$

The clinical benefit of IVC filter placement was addressed in one prospective trial (the PREPIC study) in which 400 patients with proximal DVT who were at risk for PE were randomized to receive an IVC filter (200 patients) or no filter (200 patients). Both groups were anticoagulated with LMWH or unfractionated heparin. At day 12, two (1.1\%) patients assigned to receive filters, as compared with nine $(4.8 \%)$ patients assigned to receive no filters, had symptomatic or asymptomatic PE (odds ratio [OR], 0.22; 95\% confidence interval [CI]: 0.05-0.90). However, at 2 years, $37(20.8 \%)$ patients assigned to the filter group, as compared with $21(11.6 \%)$ patients assigned to the no-filter group, had recurrent DVT (OR 1.87; 95\% CI: 1.10-3.20). ${ }^{8}$ This study was updated eight years later; patients with IVC filters experienced a greater cumulative incidence of symptomatic DVT (35.7\% versus 27.5\%; hazard ratio [HR] 1.52, CI: $1.02-2.27 ; P=0.042)$, but significantly fewer symptomatic pulmonary emboli $(6.2 \%$ versus $15.1 \%$; HR 0.37 , CI: $0.17-0.79 ; P=0.008) .{ }^{9}$ The conclusion from this

Table I Indications for IVC filter placement

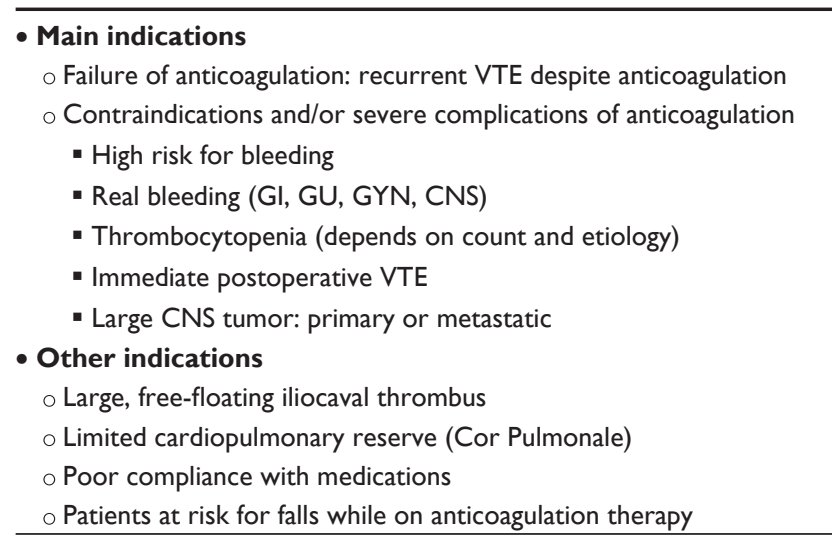

Abbreviations: IVC, inferior vena cava; GI, gastrointestinal; GU, genitourinary; GYN, gynecological; CNS, central nervous system; VTE, venous thromboembolism. 
long-term follow-up was similar to the original report; that is, with an IVC filter there is an equivalent trade-off of fewer $\mathrm{PE}$ at the cost of more DVTs. There was no difference in long-term morbidity or mortality in both groups.

Given the lack of long-term benefits of IVC filters, temporary, retrievable filters have gained increasing interest. Many different retrievable filters have recently received approval for temporary insertion. Recent data suggest that the use of these filters may be associated with low rates of PE and insertion complications. ${ }^{10}$ Nevertheless, no randomized clinical trials have been performed. In one large retrospective study that included 252 evaluable patients who had retrievable filter placed for different indications, only 47 filters were successfully retrieved, yielding a retrieval rate of $18.7 \% .{ }^{11}$ Similar or higher retrieval rates were reported by others. ${ }^{12}$

Regardless of the type of the filter placed, the most recent American Colleague of Chest Physicians (ACCP) guidelines recommend systemic anticoagulation, when possible, even with the filter in place. $^{13}$

Cancer itself, or its treatment, may result in certain clinical complications that make systemic anticoagulation very risky. Venous thromboembolic disease is a frequent complication in patients with intracranial malignancies. Many of the primary brain tumors like gliomas or secondary metastatic tumors to the brain are either bulky or very vascular, thus increasing the risk of bleeding with or without systemic anticoagulation. ${ }^{14}$ Brain metastases from melanoma, choriocarcinoma, thyroid carcinoma, and renal cell carcinoma have particularly high propensities for spontaneous hemorrhage, while metastatic tumors from sites like the lung and breast are less likely to bleed spontaneously. ${ }^{15}$ However, not all patients with intracranial malignancies are at higher risk of bleeding with anticoagulation. The complication rate of IVC filters in patients with brain tumors is higher than commonly perceived and may outweigh the risk of anticoagulation. Researchers at Brigham and Women's Hospital in Boston reviewed the records of 49 patients with intracranial malignancies and venous thromboembolic disease to determine the effectiveness and complications resulting from systemic anticoagulation or IVC filter placement. Of the 42 patients who received IVC filters, a strikingly high percentage (62\%) developed one or more complications; $12 \%$ developed recurrent PE, while $57 \%$ developed filter thrombosis, recurrent DVT, or postphlebitic syndrome. These complications severely reduced the quality of life of affected patients. Only 15 (31\%) patients were treated with anticoagulation, and seven of these received it because of continued thromboembolic disease. None of these 15 patients had proven hemorrhagic complications. ${ }^{16}$
Many recent studies questioned the need to insert IVC filters in cancer patients, particularly those with advancedstage disease whose survival is short, and prevention of PE may be of little clinical benefit and could be a poor utilization of resources. In one retrospective study performed to determine the clinical benefit of IVC filter placement in patients with malignancy, 116 patients who had such filters inserted were included. A total of 91 (78\%) patients had stage IV disease, 42 (46\%) of them died of cancer within six weeks, and only $16(14 \%)$ were alive at one year. ${ }^{17}$

The benefits of IVC filter placement on overall survival, as measured from the time of VTE, was addressed in a recent retrospective study that examined 206 consecutive cancer patients with VTE. Patients were classified into three treatment groups: anticoagulation-only $(n=62)$, IVC filter-only $(\mathrm{n}=77)$, or a combination of both IVC filter and anticoagulation $(n=67)$. Median overall survival was significantly greater in patients treated with anticoagulation (13 months) compared with those treated with IVC filters (two months) or combination of both (3.25 months; $P<0.0002)$. IVC patient's risk of death was at 1.9 times more than anticoagulation only (HR $=0.528 ; 95 \% \mathrm{CI}$ : 0.374-0.745). Multivariate analysis revealed that performance status and type of thrombus were not confounders and had no effect on overall survival. ${ }^{18}$

In another study, the survival benefit of placing IVC filters in patients with late-stage malignancy was evaluated in a group of 5970 patients who were treated with a primary diagnosis of malignancy at a tertiary care facility. Retrospective analysis identified 55 consecutive patients with stage III or IV malignant disease and VTE who received IVC filters. In a case control study, 16 patients with VTE but without IVC filter were matched for age, sex, type of malignancy, and stage of disease. Filter placement prevented $\mathrm{PE}$ in $52(94.5 \%)$ patients, however, four $(7.3 \%)$ patients had complications related to the procedure; $13(23.6 \%)$ patients with late-stage cancer survived less than 30 days following the placement of the filter; another $13(23.6 \%)$ patients of this group, however, survived more than one year. Ambulatory status differed significantly $(P=0.01)$ between these two subgroups. Authors concluded that IVC filter placement conferred no survival benefit compared with the control group and that the survival of such patients with advanced-stage cancer was limited primarily by the malignant process. ${ }^{19}$ Researchers at MD Anderson Cancer Center concluded, in a study that included 308 cancer patients with VTE and IVC filters, that such filters are safe and effective in preventing PE-related deaths in selected patients 
with cancer. However, patients with a history of DVT and bleeding or advanced disease had the lowest survival after IVC filter placement. ${ }^{20}$

\section{Conclusion}

Venous thromboembolism is commonly encountered in cancer patients. While systemic anticoagulation can be safely offered for the majority of such patients, the risk of bleeding or PE can be occasionally high. Placement of IVC filters in cancer patients should take into consideration the stage of disease and their life expectancy. While such filters can be effective in preventing PE, there may be limited survival benefit in patients with advanced-stage malignancies. Many studies have suggested that the placement of IVC filters in such patients does not improve survival and may negatively affect the quality of life. In our case under discussion, a decision was made not to insert a filter and the patient died two weeks later.

\section{Acknowledgments}

The authors would like to thank Ms Haifa Al-Ahmad and Mrs Alice Haddadin for their help in preparing this manuscript.

\section{Disclosure}

The authors declare no conflicts of interest.

\section{References}

1. Heit JA, Silverstein MD, Mohr DN, et al. Risk factors for deep vein thrombosis and pulmonary embolism: a population-based case-control study. Arch Intern Med. 2000;160:809-815.

2. Heit JA, O'Fallon WM, Petterson TM, et al. Relative impact of risk factors for deep vein thrombosis and pulmonary embolism: a population-based study. Arch Intern Med. 2002;162:1245-1248.

3. Thodiyil PA, Kakkar AK. Variation in relative risk of venous thromboembolism in different cancers. Thromb Haemost. 2002;87: 1076-1077.

4. Buller HR, Davidson BL, Decousus H, et al. Fondaparinux or enoxaparin for the initial treatment of symptomatic deep venous thrombosis: a randomized trial. Ann Intern Med. 2004;140:867-873.
5. Schwarz RE, Marrero AM, Conlon KC, Burt M. Inferior vena cava filters in cancer patients: indications and outcome. J Clin Oncol. 1996; 14:652-657.

6. Saour J, Al Harthi A, El Sherif M, Bakhsh E, Mammo L. Inferior vena caval filters: 5 years of experience in a tertiary care center. Ann Saudi Med. 2004;29:446-449.

7. Spencer FA, Bates SM, Goldberg RJ, et al. A population-based study of inferior vena cava filters in patients with acute venous thromboembolism. Arch Intern Med. 2010;170:1456-1462.

8. Decousus H, Leizorovicz A, Parent F, et al. A clinical trial of vena caval filters in the prevention of pulmonary embolism in patients with proximal deep-vein thrombosis: Prevention duRisque d'Embolie Pulmonaire par Interruption Cave Study Group. N Engl J Med. 1998; 338:409-415.

9. The PREPIC Study Group. Eight-year follow-up of patients with permanent vena cava filters in the prevention of pulmonary embolism: the PREPIC (Pre'vention du Risque d'Embolie Pulmonaire par Interruption Cave) randomized study. Circulation. 2005;112:416-422.

10. Imberti D, Prisco D. Retrievable vena cava filters: key considerations. Thromb Res. 2008;122:442-449.

11. Dabbagh O, Nagam N, Chitima-Matsiga R, Bearelly S, Bearelly D. Retrievable inferior vena cava filters are not getting retrieved: where is the gap? Thromb Res. 2010;126:493-497.

12. Mismetti P, Rivron-Guillot K, Quenet S, et al. A prospective long-term study of 220 patients with a retrievable vena cava filter for secondary prevention of venous thromboembolism. Chest. 2007;131:223-229.

13. Kearon C, Kahn SR, Agnelli G, Goldhaber S, Raskob GE, Comerota AJ; for the American College of Chest Physicians. Antithrombotic therapy for venous thromboembolic disease: American College of Chest Physicians Evidence-Based Clinical Practice Guidelines. 8th ed. Chest. 2008;133(6 Suppl):454S-545S.

14. Ruff RL, Posner JB. The incidence and treatment of peripheral venous thrombosis in patients with glioma. Ann Neurol. 1983;13:334-336.

15. Mandybur TI. Intracranial hemorrhage caused by metastatic tumors. Neurology. 1977;27:650-655.

16. Levin JM, Schiff D, Loeffler JS, Fine HA, Black PM, Wen PY. Complications of therapy for venous thromboembolic disease in patients with brain tumors. Neurology. 1993;43:1111-1114.

17. Jarrett BP, Dougherty MJ, Calligaro KD. Inferior vena cava filters in malignant disease. J Vasc Surg. 2002;36:704-707.

18. Barginear MF, Lesser M, Akerman ML, et al. Need for inferior vena cava filters in cancer patients: a surrogate marker for poor outcome. Clin Appl Thromb Hemost. 2009;15:263-269.

19. Schunn C, Schunn GB, Hobbs G, Vona-Davis LC, Waheed U. Inferior vena cava filter placement in late-stage cancer. Vasc Endovascular Surg. 2006;40:287-294.

20. Wallace MJ, Jean JL, Gupta S, et al. Use of inferior vena caval filters and survival in patients with malignancy. Cancer. 2004;101:1902-1907.
Therapeutics and Clinical Risk Management

\section{Publish your work in this journal}

Therapeutics and Clinical Risk Management is an international, peerreviewed journal of clinical therapeutics and risk management, focusing on concise rapid reporting of clinical studies in all therapeutic areas, outcomes, safety, and programs for the effective, safe, and sustained use of medicines. This journal is indexed on PubMed Central, CAS,

\section{Dovepress}

EMBase, Scopus and the Elsevier Bibliographic databases. The manuscript management system is completely online and includes a very quick and fair peer-review system, which is all easy to use. Visit $\mathrm{http}: / /$ www.dovepress.com/testimonials.php to read real quotes from published authors. 